\section{Case Reports in Oncology}

\title{
Occult Disseminated Metastatic Breast Carcinoma Presenting as Acquired Thrombotic Thrombocytopenic Purpura
}

\author{
Siew Lian Chong a,b Asral Wirda Ahmad Asnawi ${ }^{a, b} \quad$ Roszymah Hamzaha \\ Pek Kuen Liew $^{a}$ Tee Chuan Ong ${ }^{a}$ Sen Mui Tan ${ }^{a} \quad$ Kian Meng Chang ${ }^{c}$ \\ aDepartment of Hematology, Hospital Ampang, Ampang, Malaysia; ${ }^{\circ}$ Faculty of Medicine \\ and Health Sciences, Universiti Sains Islam Malaysia, Nilai, Malaysia; 'Department of \\ Medicine, Sunway Medical Centre, Petaling Jaya, Malaysia
}

\section{Keywords}

Microangiopathy - ADAMTS13 - Carcinoma - Thrombotic thrombocytopenic purpura ·

Hemolysis

\section{Abstract}

Cancer-related microangiopathic hemolytic anemia (MAHA) is a rare and life-threatening condition. We present a patient who had been treated for invasive lobular breast carcinoma in clinical remission with fever and hemolytic anemia. The peripheral blood film showed MAHA and thrombocytopenia, and a functional deficiency of ADAMTS13 activity of $23 \%$ consistent with acquired thrombotic thrombocytopenic purpura. Bone marrow aspirate and trephine biopsy confirmed metastatic carcinoma. Further evaluation revealed the involvement of multiple bone sites without recurrence of the primary tumor. The patient received a daily plasma exchange with cryosupernatant and was pulsed with corticosteroids. MAHA related to breast cancer appears to be a rare occurrence. 
Chong et al.: Breast Cancer and Thrombotic Thrombocytopenic Purpura

\section{Introduction}

Thrombotic thrombocytopenic purpura (TTP) has been classified into thrombotic microangiopathy (TMA) syndromes that encompass various clinical conditions. These conditions are unified by the pathological characteristic of vascular damage with the characteristic of endotheliosis, including lumen and vessel wall fibrin, which is manifested by arteriolar and capillary thrombosis [1]. The first reported case of TTP was in 1925 when a 16-year-old woman presented with weakness, pallor, purpura, and hemiparesis [2]. Before the precise pathology was fully understood, TTP led to death in virtually all cases described. The original triad of hemolytic anemia, thrombocytopenia, and neurological disease has expanded to include fever and renal disease. It would be nearly 60 years later when unusual large multimers of the von Willebrand factor (vWF) were observed in patients with hereditary TTP that ultimately led to the identification of the vWF-cleaving protease, ADAMTS13 (a disintegrin and metalloproteinase with a type 1 thrombospondin motif) [3]. Quantitative deficiency of ADAMTS13 leads to unusually large vWF multimers that precipitate the buildup of platelet microthrombi in small vessels and capillaries [4]. TTP can be caused by congenital deficiency of ADAMTS13 caused by homozygous or compound heterozygous mutations [3]. However, acquired TTP due to autoimmune antibodies which can neutralize or non-neutralize against ADAMTS13 activity is more commonly observed [5]. The presence of the causal abnormality may not become clinically apparent until a particular condition precipitates an acute TMA episode. Therefore, these patients are clinically distinct from most patients with microangiopathic hemolytic anemia (MAHA) and thrombocytopenia that represent manifestations of an underlying disorder. Here, we describe a patient with a history of clinical remission invasive breast carcinoma who presented fever and hemolytic anemia as the first symptoms of metastasis.

\section{Report/Case Presentation}

A 48-year-old woman presented to the emergency department with a 1-week history of fever. The fever was low grade and intermittent. It was associated with lethargy and extreme fatigue, suggesting symptomatic anemia and yellowish discoloration of the sclera. It was not associated with dyspnea, upper respiratory tract symptoms, abdominal pain, seizures, altered mental state, and changes in urinary or bowel habits. Neither apparent blood loss nor infectious causes could be detected. Physical examination at admission revealed that the patient was alert, pale, and jaundiced. Her temperature was $37.4^{\circ} \mathrm{C}$, and normotensive with mild tachycardia and good pulse volume. Thorough systemic physical examination was unremarkable. Before this, he was admitted to a district hospital for similar symptoms. She received an allogeneic blood transfusion but declined to do further investigation.

The patient had a history of primary left-breast carcinoma (T2N0M0) with a histopathological appearance of invasive carcinoma of no special type that was diagnosed 3 years earlier. Histologically, the tumor was grade II, based on well-preserved tubule formation $(3 / 3)$, the presence of pleomorphism (2/3), and mitosis (1/3). Immunohistochemistry showed that the tumor expressed progesterone and estrogen hormone receptors, while HER2 was negative. A left breast mastectomy and axillary clearance were performed. There was no nodal involvement or distant metastases. She completed 4 cycles of chemotherapy, and her last cycle was 2 years before this presentation. She received a nonsteroidal antiestrogen after chemotherapy and remained well. While attending regular follow-up with the oncology team, she was regarded as in clinical remission, and she did not have any other medical illnesses.

Her baseline hemoglobin level was $12.1 \mathrm{~g} / \mathrm{dL}$, which dropped to $7.9 \mathrm{~g} / \mathrm{dL}$ upon admission. Initial laboratory investigations showed hemolytic anemia (Table 1). An urgent peripheral

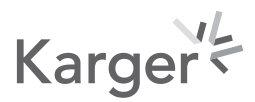




\section{Case Reports in Oncology}

Table 1. Laboratory data

\begin{tabular}{l|l}
\hline Case Rep Oncol 2021;14:1814-1820 \\
\hline DOI: 10.1159/000521159 & $\begin{array}{l}\text { ○ 2021 The Author(s). Published by S. Karger AG, Basel } \\
\text { www.karger.com/cro }\end{array}$ \\
\hline
\end{tabular}

Chong et al.: Breast Cancer and Thrombotic Thrombocytopenic Purpura

\begin{tabular}{llc}
\hline Variable & Result & Reference range \\
\hline Hemoglobin, g/dL & 6.4 & $11.6-15.1$ \\
Reticulocytes, $\%$ & 21.2 & $0.4-1.6$ \\
White cell count, $\times 10^{9} / \mathrm{L}$ & 9.1 & $4.078-11.370$ \\
Platelet count, $\times 10^{9} / \mathrm{L}$ & 75 & $171-399$ \\
Prothrombin time, s & 15.2 & $11.7-14.0$ \\
Albumin, g/L & 37 & $35-52$ \\
Alkaline phosphatase, U/L & 58 & $35-104$ \\
Alanine aminotransferase, U/L & 22 & $0-33$ \\
Total bilirubin, $\mu$ mol/L & 44.1 & $0-21$ \\
Lactate dehydrogenase, U/L & 3651 & $240-480$ \\
Creatinine & 99 & $44-80$ \\
Urea nitrogen & 8.70 & $2.76-8.07$ \\
*Glomerular filtration rate, $\mathrm{mL} / \mathrm{min}$ & 68 & \\
\hline \multicolumn{2}{c}{$*$ The glomerular filtration rate was calculated using the Cockcroft- } \\
Gault formula.
\end{tabular}

blood smear showed features of MAHA and thrombocytopenia with around 20-25 schistocytes seen per high-power field view. Immature granulocytes, atypical cells, and nucleated red cells were not seen (shown in Fig. 1a). The direct Coombs test was negative, and the coagulation screen was normal. Based on the clinical presentation and laboratory findings, a diagnosis of acquired TTP was made. Treatment was started with a pulse of intravenous methylprednisolone (25 mg/kg/day), and subsequent plasmapheresis was started. Arrangements with the transfusion unit for plasma exchange were made for 1-1.5 total blood volume with cryosupernatant. A sample was taken for quantification of ADAMTS13 activity before plasma exchange and was low at 23\% (reference range: $40-130 \%$ ). She was also supplemented with folic acid, covered with an empirical antibiotic for Gram-negative bacteria, and prophylactic treatment for thrombotic risk was started with subcutaneous low molecular weight heparin. On the second day of plasma exchange, her Hb level was $5.7 \mathrm{~g} / \mathrm{dL}$, platelet $26 \times 10^{9} / \mathrm{L}$, and LDH 1,112 U/L. Given her history, a bone marrow aspirate and trephine biopsy were performed to exclude any hematological malignancy or bone marrow infiltration. Examination of the bone marrow showed replacement of the marrow by sheets of atypical cells that form glands and signet-ring cells in nests lined by fibrovascular stroma (shown in Fig. 1b-d). These findings were consistent with metastatic carcinoma. A CT scan of the brain, thorax, abdomen, and pelvis as well as a bone scan were arranged for reassessment and restaging of her primary tumor. The CT scan showed bone metastasis to the L2 vertebra with incidental findings of liver cysts and bilateral renal cortical cysts. Lesions in the right breast parenchyma or significant right axillary lymph nodes were absent. No other lesions in the lungs or brain were seen (shown in Fig. 2a). The bone scan showed abnormal foci of increased tracer uptake at multiple sites involving the C6 vertebra body; sternum; second, ninth, and tenth right rib; fifth left rib; and vertebra body L2 (shown in Fig. 2b). A diagnosis of metastatic breast carcinoma was made and a prompt referral to the oncologist for further evaluation and treatment. The patient was followed up by the oncology unit where she was counseled for palliative support and management. When considering the maintenance of quality of life with a receptor-positive tumor, an aromatase inhibitor with adequate analgesia was prescribed. 


\section{Case Reports in Oncology}

\begin{tabular}{l|l}
\hline Case Rep Oncol 2021;14:1814-1820 \\
\hline DOI: 10.1159/000521159 & $\begin{array}{l}\text { ○ 2021 The Author(s). Published by S. Karger AG, Basel } \\
\text { www.karger.com/cro }\end{array}$ \\
\hline
\end{tabular}

Chong et al.: Breast Cancer and Thrombotic Thrombocytopenic Purpura

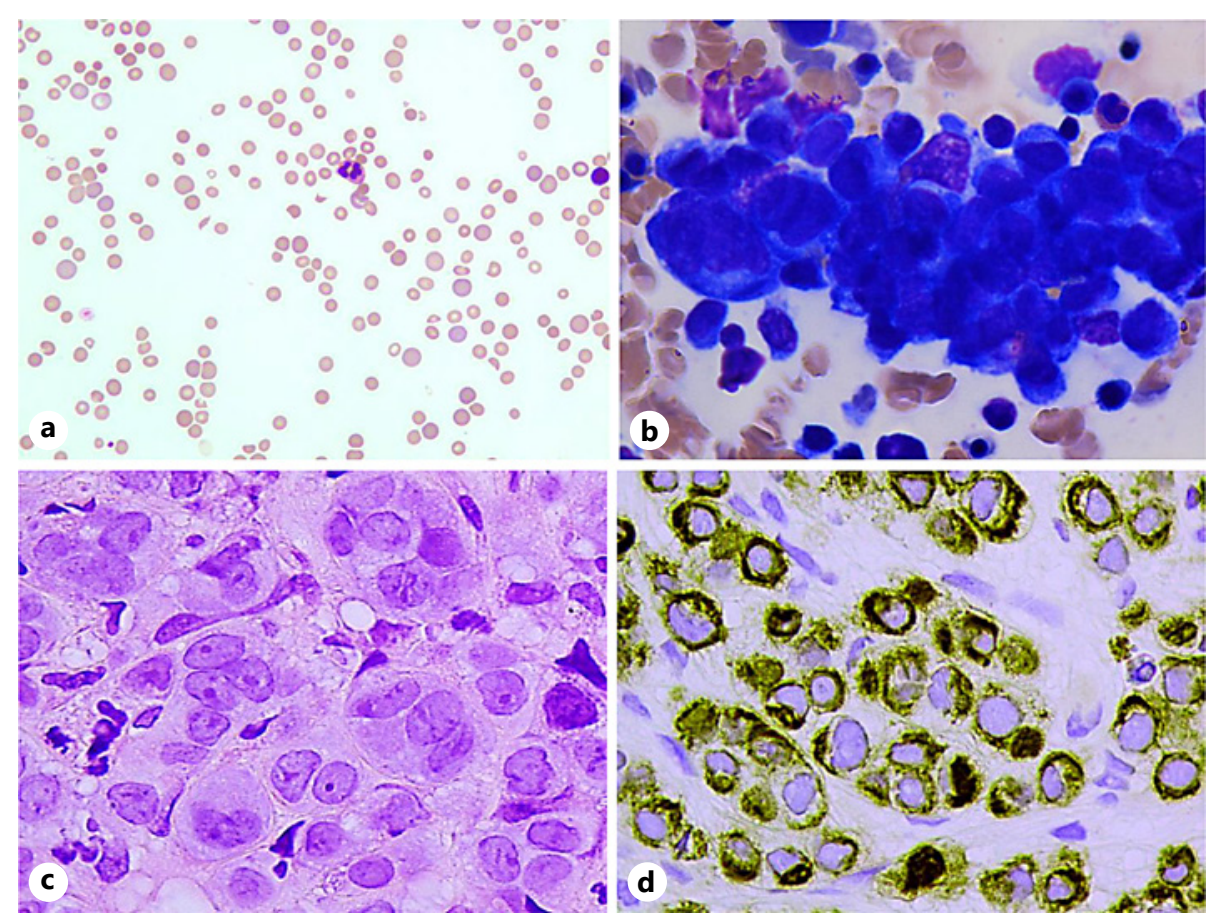

Fig. 1. a Peripheral blood film shows hemolytic anemia with polychromasia, microspherocytes, and schistocytes (Wright's stain, $\times 40$ ). $\mathbf{b}$ The bone marrow aspirate contains numerous clumps of nonhematopoietic cells (May-Grunwald-Giemsa, $\times 100$ ). c Trephine biopsy shows infiltration by sheets of nonhematopoietic cells that are moderate to large, dispersed chromatin, and prominent nucleoli in a cohesive nesting pattern $(\mathrm{H} \& \mathrm{E}, \times 100)$ and $(\mathbf{d})$ positive for cytokeratin (immunostain, $\times 100)$.
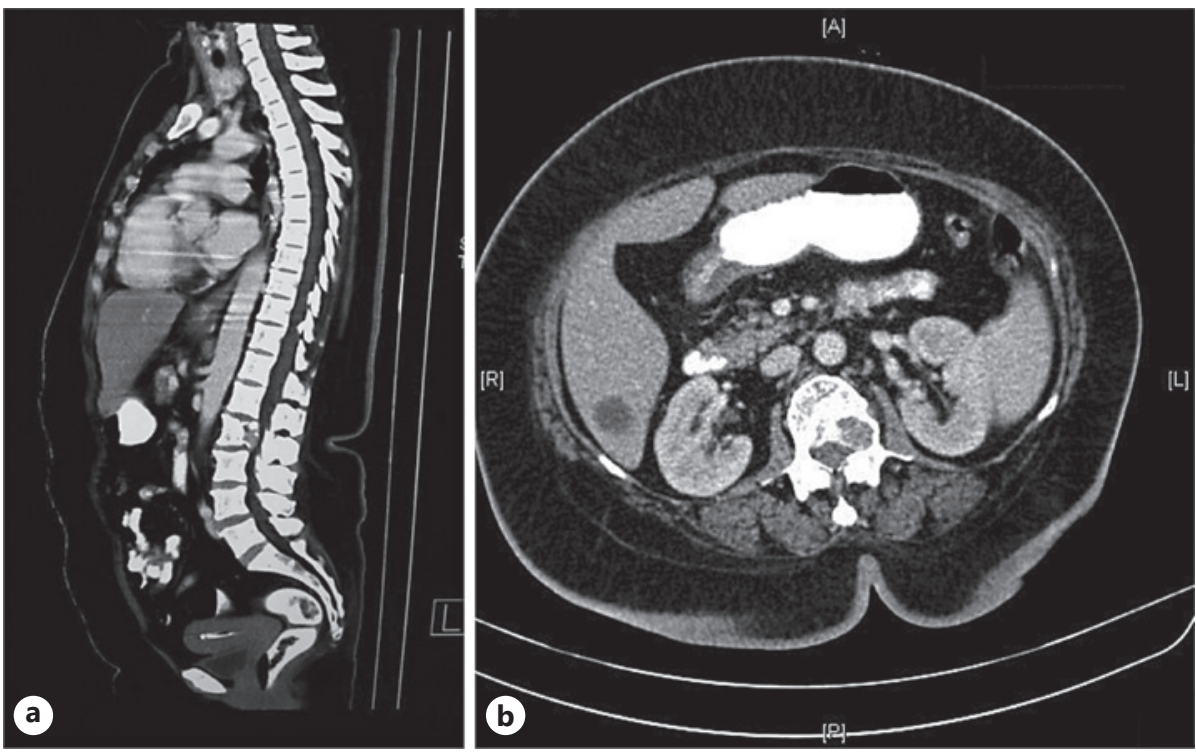

Fig. 2. a Computed tomography scan shows lytic-sclerotic lesions on the L2 vertebra body. b Bone scan shows bone metastasis to the ribs, sternum, and lumbar vertebrae. 


\section{Discussion/Conclusion}

Cancer-related MAHA was first described in 1979 in a few case series of solid tumors [6]. This condition has since been studied as single case reports and literature reviews. The pathogenesis of cancer-related MAHA remains unknown. However, several mechanisms have been suggested. The presence of tumor microemboli can cause mechanical lysis of red blood cells in the microvasculature. Circulating tumor cells can induce an inflammatory syndrome that activates endothelial cells or causes endothelial injury that triggers platelet aggregation at the site and activation of the coagulation cascade. This may be enhanced by high tissue factor expression by activated or injured endothelial cells and tumor cells, mucin-producing tumors, and vWF release caused by bone marrow metastasis [7, 8] Apart from lymphomas, gastric and breast adenocarcinomas were the 2 most common primary tumor types in patients with cancer-related MAHA [9]. Breast cancer-related MAHA is a rare paraneoplastic syndrome. This paraneoplastic syndrome in the form of TMA presents as acquired TTP, which may be the only presentation of occult disseminated malignancy without recurrence of the primary tumor. In the absence of an alternative explanation and exclusion of disseminated intravascular coagulopathy, acquired TTP would be the main impression. Acquired TTP can be distinguished into 2 types: immune-mediated forms due to an autoimmune disorder caused by the presence of inhibition of ADAMTS13 activity [10] or secondary to endothelial stimulation. In this type, massive endothelial stimulation leads to the release of ultra-large vWF multimers that exceed the body's ability to degrade them [11]. In this case, ADAMTS13 activity was reduced. However, ADAMTS13 activity is typically $<10 \%$ in patients with autoimmune-type TTP. Acquired TTPs presenting with normal or mildly reduced levels of ADAMTS13 levels $(>10 \%)$ are metastatic tumors, organ transplantation and drugs such as cyclosporine, mitomycin, and $\alpha$-interferon presence were not performed in this case. Breast cancer-related MAHA remains a poorly understood entity.

A study by Alhenc-Gelas et al. [12] examined MAHA related to breast cancer from multiple major breast cancer centers over 20 years. They reported 54 cases within that time frame, confirming the rarity of this condition; however, the incidence rate was not determined because the number of patients with metastatic breast cancer at the same time was unknown. Breast adenocarcinoma was described as the most common histology associated with MAHA was lobular or lobular histology. This histological type had many phenotypic and genotypic similarities with gastric adenocarcinomas, such as low E-cadherin [13]. Mucin expression could trigger platelet aggregation within the microvessels that is independent of tissue factor secretion [14]. Regarding the immunohistochemical profile in this patient, the hormone receptor-positive/HER2-negative subtype was consistent with poor outcomes in metastatic breast carcinoma. This case represents an atypical presentation of metastasis as TTP was the presenting complaint that urged further investigative methods to be done. However, this follows other MAHA reports in which all patients had an advanced stage of the disease, and most patients had multiple metastatic sites [7]. In line with other observations [7], acute kidney injuries and neurological manifestations were absent.

Survival of acquired TTP before the use of plasma exchange was 10\% [15]. Prompt initiation of daily therapeutic plasma exchange in conjunction with corticosteroids is required. If plasmapheresis could not be initiated, infusion with fresh frozen plasma to replace ADAMTS13 may be administered until plasma exchange can occur. Overall survival in patients with cancer-related MAHA was reported to be very poor [12]. Factors such as altered performance status, abnormal prothrombin time, and elevated total bilirubin have emerged as the strongest independent prognostic factors [12]. However, no survival prognostic factors have been identified or implemented in clinical practice. Clinical decision-making guided by prognostic factors for scoring may be useful in this serious and potentially fatal condition.

\section{Karger'}


The disseminated occult malignancy may mimic acquired TTP. High clinical suspicion and thorough investigation of the underlying malignancy are recommended, especially for TTP with atypical clinical presentation.

\section{Acknowledgment}

We would like to thank the director general of Health Malaysia for his permission to publish this article.

\section{Statement of Ethics}

The research was carried out ethically according to the Declaration of Helsinki of the World Medical Association. Since the patient had passed away at the time of writing, written informed consent was obtained from the next of kin for the publication of this case report. The completed consent form is available to the editor if requested. This is a case report; thus, the paper is exempt from Ethical Committee approval.

\section{Conflict of Interest Statement}

The authors have no conflicts of interest to declare.

\section{Funding Sources}

No research support was obtained for this report.

\section{Author Contributions}

S.L.C. and R.H. compiled the investigations and wrote the manuscript. T.C.O., K.M.C., and S.M.T. were responsible for the clinical data. P.K.L. and A.W.A.A. revised and edited the manuscript. All the authors approved the final version to be published and agreed to act as guarantors of the work.

\section{Data Availability Statement}

Additional data will be made available upon reasonable request.

\section{References}

1 Laszik Z. Hemolytic uremic syndrome, thrombotic thrombocytopenic perpura, and other thrombotic microangiopathies. Heptin Pathol Kidney. 2007;701-64.

2 Moschcowitz E. An acute febrile pleiochromic anemia with hyaline thrombosis of the terminal arterioles and capillaries: an undescribed disease. Arch Intern Med. 1925;36(1):89-93.

3 Levy GG, Nichols WC, Lian EC, Foroud T, McClintick JN, McGee BM, et al. Mutations in a member of the ADAMTS gene family cause thrombotic thrombocytopenic purpura. Nature. 2001;413(6855):488-94.

4 Moake JL. Thrombotic microangiopathies. N Engl J Med. 2002;347(8):589-600. 
5 Reese JA, Muthurajah DS, Hovinga JAK, Vesely SK, Terrell DR, George JN. Children and adults with thrombotic thrombocytopenic purpura associated with severe, acquired Adamts13 deficiency: comparison of incidence, demographic and clinical features. Pediatr Blood Cancer. 2013;60(10):1676-82.

6 Antman KH, Skarin AT, Mayer RJ, Hargreaves HK, Canellos GP. Microangiopathic hemolytic anemia and cancer: a review. Medicine. 1979;58(5):377-84.

7 Oberic L, Buffet M, Schwarzinger M, Veyradier A, Clabault K, Malot S, et al. Cancer awareness in atypical thrombotic microangiopathies. Oncologist. 2009;14(8):769-79.

8 Zwicker JI, Liebman HA, Neuberg D, Lacroix R, Bauer KA, Furie BC, et al. Tumor-derived tissue factor-bearing microparticles are associated with venous thromboembolic events in malignancy. Clin Cancer Res. 2009; 15(22):6830-40.

9 Lechner K, Obermeier HL. Cancer-related microangiopathic hemolytic anemia: clinical and laboratory features in 168 reported cases. Medicine. 2012;91(4):195-205.

10 Rieger M, Mannucci PM, Hovinga JAK, Herzog A, Gerstenbauer G, Konetschny C, et al. ADAMTS13 autoantibodies in patients with thrombotic microangiopathies and other immunomediated diseases. Blood. 2005; 106(4):1262-7.

11 van der Plas RM, Schiphorst ME, Huizinga EG, Hené RJ, Verdonck LF, Sixma JJ, et al. von Willebrand factor proteolysis is deficient in classic, but not in bone marrow transplantation-associated, thrombotic thrombocytopenic purpura. Blood. 1999;93(11):3798-802.

12 Alhenc-Gelas M, Cabel L, Berger F, Delaloge S, Frenel JS, Levy C, et al. Characteristics and outcome of breast cancer-related microangiopathic haemolytic anaemia: a multicentre study. Breast Cancer Res. 2021;23(1): $1-10$.

13 Schrader KA, Masciari S, Boyd N, Wiyrick S, Kaurah P, Senz J, et al. Hereditary diffuse gastric cancer: association with lobular breast cancer. Fam Cancer. 2008;7(1):73-82.

14 Wahrenbrock M, Borsig L, Le D, Varki N, Varki A. Selectin-mucin interactions as a probable molecular explanation for the association of Trousseau syndrome with mucinous adenocarcinomas. J Clin Invest. 2003; 112(6):853-62.

15 Amorosi EL, Ultmann JE. Thrombotic thrombocytopenic purpura: report of 16 cases and review of the literature. Medicine. 1966;45(2):139-60. 